\title{
THE SUBGROUP GROWTH SPECTRUM OF VIRTUALLY FREE GROUPS
}

\author{
JAN-CHRISTOPH SCHLAGE-PUCHTA
}

\begin{abstract}
For a finitely generated group $\Gamma$ denote by $\mu(\Gamma)$ the growth coefficient of $\Gamma$, that is, the infimum over all real numbers $d$ such that $s_{n}(\Gamma)<n !^{d}$. We show that the growth coefficient of a virtually free group is always rational, and that every rational number occurs as growth coefficient of some virtually free group.
\end{abstract}

For a finitely generated group $\Gamma$ denote by $s_{n}(\Gamma)$ the number of subgroups of index $n$ in $\Gamma$, and define the growth coefficient $\mu(\Gamma)$ as

$$
\mu(\Gamma)=\limsup _{n \rightarrow \infty} \frac{\log s_{n}(\Gamma)}{n \log n} .
$$

This quantity has been computed in a variety of cases, including free product of finite groups [5], Fuchsian groups [6], and certain one-relator groups [7]. In all these examples, $\mu(\Gamma)$ can be expressed as $-1+\sum_{i=1}^{k}\left(1-\frac{1}{n_{i}}\right)$ for certain integers $n_{i} \geq 2$. This observation led to the question as to which values $\mu(\Gamma)$ can attain; in particular, in [8] it was asked whether there exists a sequence of groups $\left(\Gamma_{n}\right)$, such that $\mu\left(\Gamma_{n}\right)$ converges from above. This question is answered by the following Theorem.

Theorem 1. The set $\{\mu(\Gamma): \Gamma$ finitely generated, virtually free $\}$ is equal to the set of non-negative rational numbers.

The proof of this result falls into two steps, the first one being the construction of virtually free groups with given growth coefficient, these groups are described in the following theorem.

Theorem 2. Let $k, \ell$ be integers, $p$ a prime number, and suppose that $k-\ell p \geq 2$. Define the group $\Gamma_{p, k, \ell}$ as the amalgamated product $S_{k}{ }^{*} C_{p} S_{k}$, where a generator of $C_{p}$ is mapped onto an element consisting of $\ell$ cycles of length $p$ in both groups. Similarly, for $p$ odd or $p=2$ and $\ell$ even, let $\Gamma_{p, k, \ell}^{+}$be the amalgamated product $A_{k} * C_{p} A_{k}$. Then we have

$$
\mu\left(\Gamma_{p, k, \ell}\right)=1-\frac{(p-1) \ell+1+\delta}{k},
$$

where $\delta=1$ if $p=2$ and $\ell$ is odd, and $\delta=0$ otherwise, apart from the exceptions $(p, k, \ell)=(2,5,2),(3,7,2)$, for which we have $\mu\left(\Gamma_{2,5,2}\right)=\frac{1}{2}$ and $\mu\left(\Gamma_{3,7,2}\right)=\frac{2}{5}$, and

$$
\mu\left(\Gamma_{p, k, \ell}^{+}\right)=1-\frac{(p-1) \ell+2}{k}
$$

with the exceptions $\mu\left(\Gamma_{2,5,2}^{+}\right)=\frac{1}{3}$ and $\mu\left(\Gamma_{3,7,2}^{+}\right)=\frac{1}{3}$.

In a second step we have to show that the growth coefficient of any virtually free group is rational. This is implied by the following result. 
Theorem 3. Let $\Gamma$ be a finitely generated virtually free group, represented by a finite graph of finite groups $\mathcal{G}$. Then the growth coefficient of $\Gamma$ is the solution of a linear optimization problem with rational coefficients which can effectively be computed from $\mathcal{G}$. In particular, $\mu(\Gamma)$ is rational.

To prove Theorem 2 we have to compute the growth coefficient of a free product with a cyclic group of prime order amalgamated, which can be done in greater generality.

Proposition 1. Let $G$ be a finite group, $x \in G$ an element of prime order $p$, and suppose that $|G|>2 p$. Set $\Gamma=G *\langle x\rangle G$, where $x$ is embedded in both copies of $G$ in the same way. Denote the conjugacy class of $x$ by $[x]$. Then $\mu(\Gamma)$ is given as

$$
\mu(\Gamma)=\frac{1}{p}+\max _{H<G}\left(\frac{(|[x] \cap H|)(p-1)}{|[x]| p}-\frac{2}{(G: H)}\right) .
$$

As another application of Proposition 1 we can compare the subgroup growth of an amalgam with its free subgroup growth. As was shown by Müller[4], the free subgroup growth is determined by the Euler characteristics, more precisely, defining $\mu_{f}(\Gamma)=\lim \sup _{n \rightarrow \infty} \frac{\log s_{n}^{f}(\Gamma)}{n \log n}$, where $s_{n}^{f}(\Gamma)$ is the number of free subgroups of index $n$ in $\Gamma$, we have for every virtually free group $\Gamma$ the relation $\mu_{f}(\Gamma)=$ $-\chi(\Gamma)$. Obviously, we always have $\mu(\Gamma) \geq \mu_{f}(\Gamma)$. For the case that $N$ is normal in both $G$ and $H$ it is shown in [9] that $\mu\left(G *_{N} H\right)=\mu((G / N) *(H / N))$, while $\chi\left(G *_{N} H\right)=\frac{1}{|G|}+\frac{1}{|H|}-\frac{1}{|N|}$ clearly depends on $N$. This leads to the question under which conditions we have $\mu(\Gamma)=-\chi(\Gamma)$. A first answer is given by the following.

Proposition 2. Let $G$ be a finite group, $x \in G$ an element of prime order $p$, and set $\Gamma=G{ }^{*} C_{p} G$, where a generator of the cyclic group is mapped onto $x$ in both copies of $G$. Then $\mu(\Gamma)=-\chi(\Gamma)$ holds true if and only if $\left|N_{G}(\langle x\rangle)\right| \leq 2 p$.

The algorithm to compute $\mu(\Gamma)$ from a graph of finite groups with fundamental group $\Gamma$ is about as difficult to perform as compiling a complete list of all subgroups of the vertex groups of the graph, which becomes a difficult problem already for relatively small groups as $S_{8}$, which has 151221 subgroups. However, if this information is available, solving the resulting linear system is quite easy.

Proposition 3. Let $\Gamma$ be the fundamental group of the graph of groups,

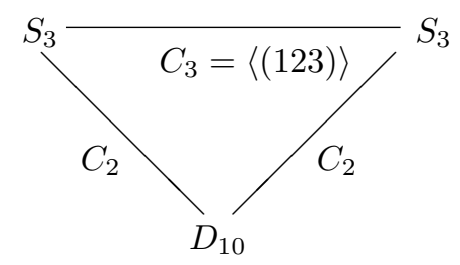

where the embeddings of $C_{2}$ identify one reflection in $D_{10}$ with the element (12) in one symmetric group, and another reflection with (13) in the other symmetric group. Then we have $\mu(\Gamma)=\frac{3}{2}$ and $\chi(\Gamma)=-\frac{9}{10}$. 
Our second example is a simpler graph of groups with a more complicated amalgamated subgroup.

Proposition 4. Let $\Gamma$ be the amalgamated product $S_{4} *_{C_{2} \times C_{2}} S_{4}$, where the subgroups $\{1,(12)(34),(13)(24),(14)(23)\}$ and $\{1,(12),(34),(12)(34)\}$ are identified. Then $\mu(\Gamma)=\frac{1}{4}$ and $\chi(\Gamma)=-\frac{1}{6}$.

\section{Some BASIC OBSERVATIONS}

Lemma 1. Let $\Gamma$ be a finitely generated group. Then we have

$$
\mu(\Gamma)=\limsup _{n \rightarrow \infty} \frac{\log \left|\operatorname{Hom}\left(\Gamma, S_{n}\right)\right|}{n \log n}-1 .
$$

Proof. Denote the right-hand side of the equation to be proven by $\mu_{h}(\Gamma)$, and let $t_{n}(\Gamma)$ be the number of homomorphisms $\Gamma \rightarrow S_{n}$ with transitive image. Then $s_{n}(\Gamma)=\frac{t_{n}(\Gamma)}{(n-1) !}$, which implies $\mu(\Gamma) \leq \mu_{h}(\Gamma)$. For the reverse inequality suppose that $\mu(\Gamma) \leq \mu_{h}(\Gamma)-\delta$ for some $\delta>0$, and let $C$ be a constant such that $s_{n}(\Gamma)<$ $C n !^{\mu_{h}(\Gamma)-\delta / 2}$ holds true for all $n \geq 1$. Let $\Omega_{1}, \ldots, \Omega_{k}$ be a partition of the set $\{1, \ldots, n\}$. Then the number of homomorphisms $\varphi: \Gamma \rightarrow S_{n}$ with image having precisely the sets $\Omega_{i}$ as orbits equals

$$
\prod_{i=1}^{k} t_{\left|\Omega_{i}\right|}(\Gamma)=\prod_{i=1}^{k}\left(\left|\Omega_{i}\right|-1\right) ! s_{\left|\Omega_{i}\right|}(\Gamma) \leq C^{k} \prod_{i=1}^{k}\left(\left|\Omega_{i}\right| !\right)^{1+\mu_{h}-\delta / 2} .
$$

Let $\lambda=\left(\lambda_{1}, \ldots, \lambda_{k}\right)$ be a partition of $n$. Then the number of homomorphisms $\varphi: \Gamma \rightarrow S_{n}$ such that the orbits of the image have sizes $\lambda_{1}, \ldots, \lambda_{k}$ equals the quantity above multiplied by the number of ways to partition a set of $n$ elements appropriately, this number in turn is bounded above by $\left(\begin{array}{c}n \\ \lambda_{1}, \ldots, \lambda_{k}\end{array}\right)$, and we find that the number of homomorphisms associated to a partition $\lambda$ is at most

$$
\left(\begin{array}{c}
n \\
\lambda_{1}, \ldots, \lambda_{k}
\end{array}\right) C^{k} \prod_{i=1}^{k}\left(\lambda_{i} !\right)^{1+\mu_{h}-\delta / 2}=n ! C^{k} \prod_{i=1}^{k}\left(\lambda_{i} !\right)^{\mu_{h}-\delta / 2} \leq n !^{1+\mu_{h}-\delta / 2} C^{k} .
$$

Since the number of partitions grows subexponentially with $n$, we deduce that the number of all homomorphisms $\varphi: \Gamma \rightarrow S_{n}$ is bounded above by $n !^{1+\mu_{h}-\delta / 2} e^{c n}$, which yields the contradiction $\mu_{h} \leq \mu_{h}-\delta / 2$.

We now show how to deduce Theorem 1 from Theorem 2 and 3. Obviously, it suffices to show that for each rational number $\frac{a}{b}$ with $a, b>0$ there exists a virtually free group $\Gamma$ with $\mu(\Gamma)=\frac{a}{b}$. Suppose first that $0<\frac{a}{b}<1$, and that $b-a$ is odd. Let $p>b$ be a prime number satisfying $(p-1, b-a)=1$. Such a prime number exists, since $b-a$ is odd, and therefore the residue class $2(\bmod b-a)$ contains infinitely many primes, each of which suffices for our purpose. Now $p-1$ is invertible modulo $b-a$, let $1 \leq \ell \leq b-a-1$ be the integer satisfying $(p-1) \ell \equiv-1(\bmod b-a)$. Finally, set $k=\frac{\bar{b} \ell(p-1)+b}{b-a}$. Provided that $\ell p \leq k-2$, we can apply Theorem 2 to find

$$
\mu\left(\Gamma_{p, k, \ell}\right)=1-\frac{(b-a)(p-1) \ell+b-a}{b \ell(p-1)+b}=\frac{a}{b} .
$$

To bound $\ell p$, note that

$$
\frac{(p-1) \ell+1}{k}=1-\frac{a}{b}
$$


thus

$$
\frac{p l}{k} \leq \frac{p(b-a)}{(p-1) b} \leq 1-\frac{p a-b}{k} \leq 1-\frac{2}{k},
$$

that is, the condition $p l \leq k-2$ is indeed satisfied.

Next, suppose that $b-a$ is even, and that $b$ is odd. Let $p>b$ be a prime number such that $(p-1, b-a)=2$, let $1 \leq \ell \leq b-a-1$ be a solution of the congruence $\ell(p-1) \equiv-2(\bmod b-a)$, and set $k=\frac{b \ell(p-1)+2 b}{b-a}$. Then we argue as above to find

$$
\mu\left(\Gamma_{p, k, \ell}\right)=1-\frac{(b-a)(p-1) \ell+2(b-a)}{b \ell(p-1)+2 b}=\frac{a}{b} .
$$

Hence, our claim holds true for all rational numbers in $(0,1)$. Now let $r$ be an integer, $\frac{a}{b} \in(0,1)$ a rational number, and $\Gamma_{a, b}$ a virtually free group with $\mu\left(\Gamma_{a, b}\right)=$ $\frac{a}{b}$. Consider the free product $\Gamma=\Gamma_{a, b} * F_{r}$. We have

$$
\left|\operatorname{Hom}\left(\Gamma, S_{n}\right)\right|=\left|\operatorname{Hom}\left(\Gamma_{a, b}, S_{n}\right)\right| \cdot\left|\operatorname{Hom}\left(F_{r}, S_{n}\right)\right|=n !^{r}\left|\operatorname{Hom}\left(\Gamma_{a, b}, S_{n}\right)\right|,
$$

thus $\mu(\Gamma)=r+\mu\left(\Gamma_{a, b}\right)=r+\frac{a}{b}$, and we find virtually free groups with growth coefficient $\frac{a}{b}$ for all non-integral positive rational numbers. Finally, all integral growth coefficients are realized by free groups, and our theorem is proven.

\section{Proof of Theorem 3}

Lemma 2. For integers $m \leq n$ we have $m !=n !^{n / m} e^{\mathcal{O}(n)}$.

Proof. By Stirling's formula we have

$$
m !=m^{m} e^{\mathcal{O}(m)}=\left(n^{n}\right)^{m / n}(m / n)^{m} e^{\mathcal{O}(m)}=n !^{m / n}(m / n)^{m} e^{\mathcal{O}(m)},
$$

thus, it suffices to show that $m \log n / m=\mathcal{O}(m)$. Differentiating with respect to $m$ shows that the left hand side is maximal for $m=n / e$, and our claim follows.

To every finitely generated virtually free group $\Gamma$ we can associate a finite graph $\mathcal{G}$ of finite groups, that is, a connected directed graph $(V, E)$, such that for each edge $e=(x, y)$ the reverse edge $\bar{e}=(y, x)$ is in $E$, together with a collection of finite groups $G_{v}, G_{e}, v \in V, e \in E$ satisfying $G_{e}=G_{\bar{e}}$ and embeddings $\psi_{e}: G_{e} \rightarrow G_{v}$ for each edge $e$ with target $v$. We define the fundamental group of $\mathcal{G}$ as follows: Let $T$ be a spanning tree of the underlying graph with all reverse edges added. Then let $\Gamma$ be the group generated by all $G_{v}, v \in V$ and symbols $g_{e}$ for all $e \in E$ subject to the relations

(1) $g_{e}=1$ for all $e \in T$,

(2) $g_{e}=g_{\bar{e}}$ for all $e \in E$,

(3) $g_{e} \psi_{e}(a) g_{e}^{-1}=\psi_{\bar{e}}(a)$ for all $e \in E, a \in G_{e}$.

We remark without proof that the fundamental group does not depend on the choice of the tree $T$, and that every finitely generated virtually finite group occurs as fundamental group of some finite graph of finite groups, for these and related results we refer the reader to [10].

Note that each vertex group is embedded in $\Gamma$, thus, each representation $\varphi: \Gamma \rightarrow$ $S_{n}$ induces representations $\varphi_{v}: G_{v} \rightarrow S_{n}$. On the other hand, not all tuples of representations $\varphi_{v}: G_{v} \rightarrow S_{n}$ can be lifted to representations of the whole group. For each tuple of representations $\left\{\varphi_{v}: G_{v} \rightarrow S_{n}, v \in V\right\}$, and every edge $e=(x, y)$, the embeddings $\psi_{e}: G_{e} \rightarrow G_{x}, \psi_{\bar{e}}: G_{e} \rightarrow G_{y}$ induce two representations of $G_{e}$, which obviously have to coincide to allow for a lift. On the other hand, it is obvious from the list of relations given above, that every tuple of representations of the $G_{v}$ 
satisfying these conditions lift to representation of $\Gamma$; in this case we will call the tuple $\left(\varphi_{v}: G_{v} \rightarrow S_{n}, v \in V\right)$ admissible. Note that admissibility only depends on the equivalence classes of the representations $\varphi_{v}$; we will refer to these data as the representation type of the tuple. The number of different equivalence classes of permutation representations of degree $n$ of a finite group equals the number of nonnegative integral solution $\left(x_{1}, \ldots, x_{m_{v}}\right)$ of the equation $\sum_{i=1}^{m_{v}} x_{i} n_{v, i}=d$, that is, the number of lattice points in a certain domain, which is contained in $[0, n]^{m_{v}}$. In particular, the number of different equivalence classes grows at most polynomially, and the value of $\mu(\Gamma)$ does not change if we replace the number of all permutation representations by the maximal number of permutation representations of $\Gamma$ with prescribed representation type. Hence, to compute $\mu(\Gamma)$ we have to determine among all admissible representation types the one leading to the greatest number of representations, and to compute this number.

Permutation representations of $G_{v}$ are direct sums of transitive representations, which in turn are equivalent to the coset action of $G_{v}$. For each $v \in V$, we therefore consider a complete list of transitive permutation representations $\rho_{v, i}, 1 \leq i \leq m_{v}$ of $G_{v}$, and let $n_{v, i}$ be the number of points $\rho_{v, i}$ acts upon. Then the equivalence class of $\varphi_{v}: G_{v} \rightarrow S_{n}$ is determined by the multiplicities $\left(\xi_{v, i}, 1 \leq i \leq m_{v}\right)$ with

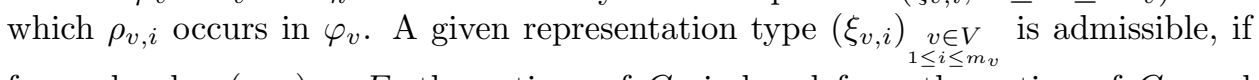
for each edge $(x, y) \in E$, the actions of $G_{e}$ induced from the action of $G_{x}$ and of $G_{y}$ coincide. To check this, note that each transitive action of $G_{x}$ gives rise to a permutation representation of $G_{e}$, which in turn decomposes into transitive $G_{e}$-actions. Hence, if $\rho_{e, i}, 1 \leq i \leq m_{e}$ is a complete list of transitive permutation representations of $G_{e}$, the multiplicities of the transitive actions of $G_{e}$ in the action induced from $G_{x}$ is the image of the vector $\left(\xi_{x, i}\right)_{1 \leq i \leq m_{x}}$ under a certain linear map given by a $m_{e} \times m_{x}$-matrix with non-negative integral entries, and the condition of admissability becomes a system of $m_{e}$ linear equations between the $m_{x}+m_{y}$ variables $\xi_{x, i}, \xi_{y, j}, 1 \leq i \leq m_{x}, 1 \leq j \leq m_{y}$.

Hence, we have shown that admissability of a representation is equivalent to a certain set of linear equations with integral coefficients in the variables $\left(\xi_{v, i}\right)_{\substack{v \in V \\ 1 \leq i \leq m_{v}}}$.

Next, we compute the number of representations for a given admissible representation type. To do so, we first choose representations of the finite groups $G_{v}$. For each $i \leq m_{v}$ we have to choose $\xi_{v, i}$ orbits of size $n_{v, i}$, the number of ways to do so is

$$
\frac{n !}{\prod_{i=1}^{m_{v}}\left(\xi_{v, i}\right) ! n_{v, i} ! \xi_{v, i}} .
$$

An action of $G_{v}$ similar to $\rho_{v, i}$ is equivalent to the action of $G_{v}$ on the cosets of a subgroup $U_{i}$ of index $n_{v, i}$. Hence, to construct an action of $G_{v}$ similar to $\rho_{v, i}$ on a given set of $n_{v, i}$ points, we have to identify these points with the cosets $G_{v} / U_{v, i}$. Two identifications give the same representation if and only if one is obtained from the other by shifting the cosets by some element $g \in G_{v}$, that is, to count different actions we have to fix one point which we identify with the coset $U_{v, i}$ itself. Hence, there are $\left(n_{v, i}-1\right)$ ! different actions equivalent to $\rho_{v, i}$, and we conclude that the number of representations of $G_{v}$ of the given type equals

$$
\frac{n !}{\prod_{i=1}^{m_{v}}\left(\xi_{v, i}\right) ! n_{v, i}^{\xi_{v, i}}} \text {. }
$$


We assume that the representation type is admissible, thus, for each edge $e$ there exist $g_{e}$ such that relations of type (3) hold. However, the relations of the first type require that the edge groups do not act similarly, but are actually equal. Hence, the number of representations of the fundamental group of the graph of groups consisting only of the vertices in $T$ with given representation type equals

(2) $\prod_{v \in V} \frac{n !}{\prod_{i=1}^{m_{v}}\left(\xi_{v, i}\right) ! n_{v, i}^{\xi_{v, i}}} \prod_{(x, y) \in T}\left(\frac{n !}{\prod_{i=1}^{m_{e}} \ell_{e, i}\left(\xi_{x, 1}, \ldots, \xi_{x, m_{v}}\right) ! n_{e, i}^{\ell_{e, i}\left(\xi_{x, 1}, \ldots, \xi_{x, m_{v}}\right)}}\right)^{-1}$,

where $\ell_{e, i}$ is the linear form giving the multiplicity of $\rho_{e, i}$ in the restriction of $\sum_{i=1}^{m_{v}} \xi_{v, i} \rho_{v, i}$ to $G_{e}$. Obviously, $\ell$ is a positive linear form with integral coefficients.

Finally, we have to choose the image of $g_{e}, e \in E \backslash T$. To do so we have to respect relations of the third type, that is, the number of possibilities to choose $g_{e}$ for $e$ equals the centralizer of a representation of $G_{e}$ of the type induced by the type of $G_{x}$. This number equals

$$
\prod_{i=1}^{m_{e}} \ell_{e, i}\left(\xi_{x, 1}, \ldots, \xi_{x, m_{v}}\right) ! n_{e, i}^{\ell_{e, i}\left(\xi_{x, 1}, \ldots, \xi_{x, m_{v}}\right)}
$$

thus the total number of representations of $\Gamma$ with representation type $\left(\xi_{v, i}\right)_{\substack{v \in V \\ 1 \leq i \leq m_{v}}}$ equals

$n !^{|E \backslash T|} \prod_{v \in V} \frac{n !}{\prod_{i=1}^{m_{v}}\left(\xi_{v, i}\right) ! n_{v, i}^{\xi_{v, i}}} \prod_{(x, y) \in E}\left(\frac{n !}{\prod_{i=1}^{m_{e}} \ell_{e, i}\left(\xi_{x, 1}, \ldots, \xi_{x, m_{v}}\right) ! n_{e, i}^{\ell_{e, i}\left(\xi_{x, 1}, \ldots, \xi_{x, m_{v}}\right)}}\right)^{-1}$.

We can now apply Lemma 2 together with the fact that the number of edges in $T$ equals $|V|-1$ to see that the logarithm of this quantity is equal to

$$
n \log n\left(1-\sum_{v \in V} \sum_{i=1}^{m_{v}} \xi_{v, i} / n+\sum_{(x, y) \in E} \ell_{e, i}\left(\xi_{x, 1}, \ldots, \xi_{x, m_{v}}\right) / n\right)+\mathcal{O}(n) .
$$

We are looking for integers $\xi_{v, i}$, such that this expression becomes maximal. To do so, we first determine real numbers $\alpha_{v, i}$ subject to conditions $\alpha_{v, i} \geq 0, \sum_{i=1}^{m_{v}} \alpha_{v, i}=$ 1 , and the equations implying admissability, such that the linear form

$$
-\sum_{v \in V} \sum_{i=1}^{m_{v}} \alpha_{v, i}+\sum_{(x, y) \in E} \ell_{e, i}\left(\alpha_{x, 1}, \ldots, \alpha_{x, m_{v}}\right)
$$

becomes maximal. This optimization problem can be solved using only matrix operations, hence, the solution is rational, since both the linear constraints as well as the function to be maximized are rational. Let $m$ be the least common multiple of all denominators occurring in the optimum, and let $\mu_{0}$ be the value of the problem. If $n$ is divisible by $m$, this rational solution gives rise to an integral solution for the $\xi_{v, i}$, and therefore to the right number of representations, which gives the lower bound $\mu(\Gamma) \geq \mu_{0}$. On the other hand, for integral values of $\xi_{v, i}$, the value of the linear problem is always bounded by the maximum over all real values, and we deduce $\mu(\Gamma)=\mu_{0}$, which proves theorem 3 . 


\section{Proof of Propositions 1 And 2}

Lemma 3. Let $G$ be a finite group, $x \in G$ an element of order $p, U<G$ a subgroup, $\varphi: G \rightarrow S_{(G: U)}$ the coset representation associated to $U$. Then the multiplicity of the trivial representation in the restriction of $\varphi$ to $\langle x\rangle$ is $\frac{|G| \cdot|[x] \cap U|}{|U||[x]|}$.

Proof. A coset $a U$ is fixed by $x$ if and only if $x^{a} \in U$. For each element in $U$ conjugate to $x$, there are $C_{G}(x)$ possible $a$ conjugating $x$ onto this element, and there are $|U|$ different $a$ defining the same coset, hence our claim.

Proof of Proposition 1. Since the amalgamated subgroup has only two inequivalent transitive permutation representations, the condition of admissability reduces to the condition that the number of fixed points of the reduced actions coincide. Hence, denoting by $U_{1}, \ldots, U_{m}$ a complete list of subgroups of $G$, a representation type $\left(\xi_{i, j}\right)_{1 \leq i \leq 2}$ is admissible if and only if

$$
\sum_{j=1}^{m} \xi_{1, j} \frac{|G| \cdot\left|[x] \cap U_{j}\right|}{\left|U_{j}\right||[x]|}=\sum_{j=1}^{m} \xi_{2, j} \frac{|G| \cdot\left|[x] \cap U_{j}\right|}{\left|U_{j}\right||[x]|} .
$$

Since there is only one edge we remove the subscript $e$ from the linear function $\ell_{e, i}$ used in (2), as well as the subscript $x$ from the variables $\xi_{x, i}$. Then $\ell_{1}\left(\xi_{1}, \ldots, \xi_{m}\right)$ equals the number of trivial representations in the restriction of a representation of $G$ similar to $\bigoplus_{i=1}^{m} \xi_{i} \cdot \rho_{i}$ to $C_{p}$. By the previous Lemma we obtain

$$
\ell_{1}\left(\xi_{1}, \ldots, \xi_{m}\right)=\sum_{i=1}^{m} \frac{|G| \cdot\left|[x] \cap U_{i}\right|}{\left|U_{i}\right||[x]|} \xi_{i} .
$$

Since the degree of the representation does not change when restricting it to a subgroup, and $C_{p}$ has only two transitive representations, we obtain

$$
\ell_{2}\left(\xi_{1}, \ldots, \xi_{m}\right)=\frac{1}{p}\left(\sum_{i=1}^{m} n_{i} \xi_{i}-\ell_{1}\left(\xi_{1}, \ldots, \xi_{m}\right)\right),
$$

since $\sum_{i=1}^{m} n_{i} \xi_{i}=n$ we deduce

$$
\ell_{1}\left(\xi_{1}, \ldots, \xi_{m}\right)+\ell_{2}\left(\xi_{1}, \ldots, \xi_{m}\right)=\frac{n}{p}+\frac{p-1}{p}\left(\sum_{i=1}^{m} \frac{|G| \cdot\left|[x] \cap U_{i}\right|}{\left|U_{i}\right||[x]|} \xi_{i}\right) .
$$

The summand $\frac{n}{p}$ clearly does not influence the optimization. The logarithm of the first factor in (2) becomes

$$
n \log n-\log n \sum_{i=1}^{m} \xi_{i}+\mathcal{O}(n)=\log n \sum_{i=1}^{m}\left(n_{i}-1\right) \xi_{i}+\mathcal{O}(n),
$$

hence, the linear form to be maximized is given as

$$
f\left(\alpha_{1,1} \ldots, \alpha_{2, m}\right)=\sum_{j=1}^{m}\left(\alpha_{1, j}+\alpha_{2, j}\right)\left(1-\frac{1}{\left(G: U_{j}\right)}\right)+\frac{p-1}{p} \sum_{j=1}^{m} \alpha_{1, j} \frac{|G| \cdot\left|[x] \cap U_{j}\right|}{\left|U_{j}\right||[x]|},
$$

our aim is to show that the maximum is attained in a point satisfying $\alpha_{1, j_{0}}=\alpha_{2, j_{0}}=$ $1, \alpha_{i, j}=0$ for $j \neq j_{0}$ for some $j_{0}$. Note first that if in the second summand of the definition of $f$ we replace all $\alpha_{1, j}$ by $\alpha_{2, j}$, the value of $f$ does not change in view of (3), and the first summand is of the form $g\left(\alpha_{1,1}, \ldots, \alpha_{1, m}\right)+g\left(\alpha_{2,1}, \ldots, \alpha_{2, m}\right)$ for a linear form $g$, thus, we may assume without loss that $\alpha_{1, j}=\alpha_{2, j}$ for all $j$. 
Under this assumption (3) is trivially satisfied. Thus, we consider the maximum of a linear form on the set $\left\{\left(\beta_{1}, \ldots, \beta_{m}\right): \beta_{i} \geq 0, \sum \beta_{i}=1\right\}$, which is either 0 , or attained in a vertex $(0, \ldots, 0,1,0, \ldots, 0)$. In the latter case our claim is proven, whereas in the former we would have $\mu(\Gamma)=0$. However, as Müller [4] showed, the number of free subgroups of $\Gamma$ of index $n|G|$ is $n !^{-\chi(\Gamma)|G|+o(1)}$. In general, the Euler characteristics of an amalgamated product $G *_{U} H$ is given by $\frac{1}{|G|}+\frac{1}{|H|}-\frac{1}{|U|}$, and, using the assumption $|G| \geq 3 p$, we obtain the contradiction

$$
0=\mu(\Gamma) \geq \frac{-\chi(\Gamma)}{|G|}=\frac{1}{p|G|}-\frac{2}{|G|^{2}} \geq \frac{1}{3 p|G|} .
$$

Proof of Proposition 2. The Euler characteristics of $G *_{C_{p}} G$ equals $-\frac{1}{p}+\frac{2}{|G|}$. Set $H=C_{p}$ in Proposition 1 to obtain

$$
\mu(\Gamma) \geq \frac{1}{p}+\frac{|[x] \cap\langle x\rangle|(p-1)}{|[x]| p}-\frac{2 p}{|G|} .
$$

Now $|[x] \cap\langle x\rangle|=\left(N_{G}(\langle x\rangle): C_{G}(x)\right)$, and $|[x]|=\left(G: C_{G}(x)\right)$, thus $\mu(\Gamma)=-\chi(\Gamma)$ implies

$$
\frac{\left(N_{G}(\langle x\rangle): C_{G}(x)\right)(p-1)}{\left(G: C_{G}(x)\right) p} \leq \frac{2 p-2}{|G|},
$$

which is equivalent to $\left|N_{G}(\langle x\rangle)\right| \leq 2 p$. To prove the converse, suppose that $\left|N_{G}(\langle x\rangle)\right| \leq 2 p$, and that the subgroup $H$ of $G$ maximizing the right-hand side of (1) is not trivial. Since every subgroup not containing an element conjugate to $x$ cannot maximize this expression, we have in particular that $|H| \geq p$. Suppose first that $p \geq 3$. Then $\left|N_{G}(\langle x\rangle)\right|<p^{2}$, thus a $p$-Sylow subgroup $P$ of $G$ is cyclic of order $p$. For suppose otherwise. Then either $x$ is central in $P$, which would imply $\left|C_{G}(\langle x\rangle)\right| \geq|P| \geq p^{2}$, or $x$ is not central in $P$, in which case there is a central element $y \in P \backslash\langle x\rangle$, which would imply $\left|C_{G}(\langle x\rangle)\right| \geq|\langle x, y\rangle| \geq p^{2}$. Hence, in each $p$-Sylow subgroup of $H$, there are $|[x] \cap\langle x\rangle|=\left(N_{G}(\langle x\rangle):\langle x\rangle\right)$ elements conjugate to $x$. Similarly, if $p=2$ and $\left|C_{H}(x)\right|$ is cyclic of order 2 or 4 , there is one conjugate of $x$ in every 2-Sylow subgroup of $H$. The number of $p$-Sylow subgroups of $H$ is $|H| / p$ at most, thus

$$
|[x] \cap H| \leq \frac{|H|}{p}|[x] \cap\langle x\rangle| \leq \frac{|H|}{p}\left(N_{G}(\langle x\rangle):\langle x\rangle\right),
$$

and the right hand side of (1) is bounded above by

$$
\begin{aligned}
\frac{1}{p}+\frac{|H|\left(N_{G}(\langle x\rangle):\langle x\rangle\right)(p-1)}{\left(G: C_{G}(\langle x\rangle)\right) p^{2}}-\frac{2}{(G: H)} & \leq \frac{1}{p}+\frac{2|H|(p-1)}{|G| p}-\frac{2|H|}{|G|} \\
& \leq \frac{1}{p}+\frac{4(p-1)}{|G|}-\frac{4 p}{|G|}
\end{aligned}
$$

since $\frac{p-1}{p}<2$ and $|H| \geq 2 p$.

It remains to consider the case $p=2, C_{H}(x) \cong C_{2} \times C_{2}$. Since $C_{H}(x) \leq N_{G}(\langle x\rangle)$, and $\left|N_{G}(x)\right| \leq 2 p=4$, we deduce $N_{G}(\langle x\rangle)=C_{H}(x)$, which implies $\left|C_{G}(x)\right|=4$. We therefore obtain $|[x] \cap H| \leq|[x]|=\frac{|G|}{4}$, and with this bound the right hand side of (1) becomes

$$
\frac{1}{2}+\frac{|H| / 4}{2|G| / 4}-\frac{2}{(G: H)}=\frac{1}{2}-\frac{3|H|}{2|G|} \leq \frac{1}{2}-\frac{6}{|G|}<-\chi(\Gamma)
$$


and the proposition is proven.

\section{Proof of Theorem 2}

We now prove Theorem 2. We have to consider 3 cases, depending on whether $x$ is an odd permutation or not, and whether we consider $S_{k} *_{C_{p}} S_{k}$ or $A_{k} *_{C_{p}} A_{k}$. Note that in the latter case $x$ is necessarily even, which is the reason we do not have 4 cases. The computations are the same for all these cases, we only give them here for the case $S_{k} *_{C_{p}} S_{k}$ and $x$ even, that is, $p \neq 2$ or $p=2$ and $\ell$ even.

To apply Proposition 1 , we have to consider the maximum taken over all subgroups of $S_{k}$. We claim that this maximum occurs for $H=A_{k-1}$, except for certain small values of $k$ which lead to the exceptions in the theorem. In this case we have $\frac{2}{S_{k}: A_{k-1}}=\frac{1}{k}$, and $|[x] \cap H|$ equals the number of elements with $\ell p$-cycles in $S_{k-1}$, thus, we obtain

$$
\frac{(|[x] \cap H|)}{|[x]|}=\frac{\frac{(k-1) !}{(k-\ell p-1) ! \ell ! p^{\ell}}}{\frac{k !}{(k-\ell p) ! ! ! p^{\ell}}}=\frac{k-\ell p}{k},
$$

and therefore the value

$$
\frac{1}{p}+\frac{(k-\ell p)(p-1)}{p k}-\frac{1}{k}=1-\frac{\ell(p-1)+1}{k} .
$$

We want to show that for every other subgroup we obtain a smaller value, that is, we have to show that for each $H<S_{k}$ different from $A_{k-1}$ we have

$$
\frac{(|[x] \cap H|)(p-1)}{|[x]| p}-\frac{2}{\left(S_{k}: H\right)}<\frac{(k-\ell p)(p-1)}{p k}-\frac{1}{k} .
$$

We will do so in four steps: First, we consider intransitive subgroups, then transitive, but imprimitive subgroups. In both cases we know that $H$ is contained in some direct product or some wreath product, and these structures are simple enough to give upper bounds for $|[x] \cap H|$. Next, we consider primitive subgroups. We know on one hand, that a proper primitive subgroup cannot be too large, on the other hand, we can show by direct construction that $x$ cannot have too many fixed points, thus, $|[x]|$ has to be large. Comparing these estimates we obtain a contradiction, provided that $n \geq 24$. Finally, we check all primitive groups of order 24 using GAP $[1]$. The quantity $\frac{([x] \cap H \mid)}{|[x]|}$ has a probabilistic interpretation as follows: Choose a permutation $\pi \in[x]$ at random. Then the probability for the event $\pi \in H$ equals $\frac{(|[x] \cap H|)}{\|x\| \mid}$. It turns out that this interpretation is useful for computing upper bounds.

An in transitive subgroup which does not contain $A_{k-1}$ has an invariant set $A$ with $2 \leq|A| \leq n / 2$, thus we have to estimate the probability that a permutation with $\ell$ cycles of length $p$ leaves a given set $A$ of size $m$ invariant. Denote this probability with $P(k, \ell, m)$. Distinguishing the possibilities that a given $p$-cycle is totally inside or outside $A$, we obtain the recursion relation

$$
P(k, \ell, m) \leq\left(\frac{m}{k}\right)^{p} P(k-p, \ell-1, m-p)+\left(1-\frac{m}{k}\right)^{p} P(k-p, \ell-1, m),
$$

which is valid for $k \geq p$ and $\ell \geq 1$. We use this relation to prove $P(k, \ell, m)<\frac{k-\ell p}{k}$ for all $m$ in the range $2 \leq m \leq k / 2$ by induction on $k$. Inserting the induction 
hypothesis we have to prove the estimate

$$
\frac{k-\ell p}{k}>\left(\frac{m}{k}\right)^{p} \frac{k-\ell p}{k-p}+\left(1-\frac{m}{k}\right)^{p} \frac{k-\ell p}{k-p},
$$

that is

$$
1-\frac{p}{k}>\left(\frac{m}{k}\right)^{p}+\left(1-\frac{m}{k}\right)^{p}
$$

The expression on the right hand side is monotonically decreasing in $m \leq k / 2$, thus, its maximal value is at $m=2$, and we see that it suffices to prove

$$
1-\frac{p}{k}>\left(\frac{2}{k}\right)^{p}+\left(1-\frac{2}{k}\right)^{p}
$$

Suppose that $k \geq 2 p$. Then the terms in the binomial expansion of $(1-2 / k)^{p}$ are alternating and of decreasing modulus, thus we obtain an upper bound by deleting all terms except the first three, and find that we only have to prove

$$
1-\frac{p+1}{k}>\left(\frac{2}{k}\right)^{p}+1-\frac{2 p}{k}+\frac{2 p(p-1)}{k^{2}}
$$

However, bounding the first summand on the right hand side by $\frac{4}{k^{2}}$, and using the assumption $k>2 p$, this inequality is seen to hold true with the possible exceptions $p=2, k=4$, which can be checked directly. Hence, the induction step is correct for $k \geq 2 p$. For $k<2 p$ we have $\ell=1$, in which case we compute $P(k, \ell, m)$ directly to obtain

$$
\begin{aligned}
P(k, 1, m) & =\frac{(k-m)(k-m-1) \cdots(k-m-p+1)}{k(k-1) \cdots(k-p+1)} \\
& \leq \frac{(k-2)(k-3) \cdots(k-p-1)}{k(k-1) \cdots(k-p+1)} \\
& =\frac{(k-p)(k-p-1)}{k(k-1)} \\
& <\frac{k-p}{k} .
\end{aligned}
$$

Hence, for intransitive subgroups our claim is proven.

Next, we consider imprimitive subgroups. Without loss we may assume that $H=S_{a} \prec S_{b}$, and denote by $\pi: H \rightarrow S_{b}$ the canonical projection.

We begin by considering some special cases, the first being the case $p=b$. If $x \in H$, then either some $S_{k}$-conjugate of $x$ contains a cycles of length $p$, which contradicts the assumption $\ell p<k$, or there are $p$ domains of imprimitivity, each of which is an invariant set under each element of $[x] \cap H$, and we can argue as in the case of intransitive groups.

Next, suppose that $a=p=2$, and denote by $P(b, \ell)$ the probability that a random element $x \in S_{k}$ consisting of $\ell$ cycles of length 2 is contained in $S_{2} \prec S_{b}$. Choose a domain of imprimitivity. Then this domain is either fixed pointwise, or a 2-cycle of $x$, or there are two 2-cycles of $x$ mapping this domain onto another domain of imprimitivity. These cases are mutually exclusive, thus we can compute 
the probability of each single case and obtain for $\ell \geq 2$ the recursion relation

$$
\begin{aligned}
& P(b, \ell)= \frac{(2 b-2 \ell)(2 b-2 \ell-1)}{2 b(2 b-1)} P(b-1, \ell) \\
&+\frac{1}{2 b-1}\left(1-\frac{(2 b-2 \ell)(2 b-2 \ell-1)}{2 b(2 b-1)}\right) P(b-1, \ell-1) \\
&+\frac{1}{2 b-1}\left(1-\frac{(2 b-2 \ell)(2 b-2 \ell-1)}{2 b(2 b-1)}\right) \\
& \quad \times\left(1-\frac{(2 b-2 \ell-2)(2 b-2 \ell-3)}{(2 b-2)(2 b-3)}\right) P(b-2, \ell-2)
\end{aligned}
$$

where $P(b-1, \ell)$ has to be interpreted as 0 if $b=\ell$. Estimating all probabilities on the right hand side as well as the brackets $(1-\ldots)$ by 1 , we obtain the estimate $P(b, \ell)<\frac{(2 b-2 \ell)(2 b-2 \ell-1)+4 b}{2 b(2 b-1)}$, which implies $P(b, \ell)<\frac{2 b-2 \ell-1}{2 b}$, and hence our claim, provided that $4 b \ell+1>6 b+4 \ell^{2}$, which is the case for all $\ell$ unless one of $b \leq 7$, $\ell \leq 1$ or $\ell \geq b-1$ holds true. For $\ell=1$ we immediatelly see that $P(b, 1)=\frac{1}{2 b-1}$, which is small enough for all $b \geq 3$. For $b=\ell$ we have

$$
P(b, b)=\frac{1}{2 b-1}(P(b-1, b-1)+P(b-2, b-2)),
$$

which implies by a simple induction that $P(b, b)<\frac{1}{b^{2}}$ for all $b \geq 6$. For $P(b, b-1)$ the recursion implies

$$
P(b, b-1)<\frac{1}{b(2 b-1)} P(b-1, b-1)+\frac{1}{2 b-1}(P(b-1, b-2)+P(b-2, b-3)) .
$$

Explicite computation implies that $P(6,5)<0.014$ and $P(7,6)<0.0051$, together with the bound for $P(b, b)$ we obtain by induction that $P(b, b-1)<\frac{1}{b^{2}}$ for all $b \geq 6$, which is again acceptable. Thus, our claim is proven for all $b \geq 8$, and for the remaining values of $b$ it can be checked directly using the recurrence relation above.

Now we embark on the general case. Let $x \in S_{k}$ be an element with $\ell$ cycles of length $p$ chosen at random; we estimate the probability that $x \in H$. We distinguish two cases, depending on whether there exists a domain of imprimitivity which is left invariant by $x$ or not. It suffices to show that in each case the conditional probability for the event $x \in H$ is at most $\frac{k-\ell p-1}{k}$. Suppose that $x$ moves some domain of imprimitivity. Then $x$ has $a$ cycles of length $p$, all of which are mapped onto the same element under $\pi$; leaving away all other restrictions on $x$ we obtain an upper bound for the probability in question. We get an even coarser bound, if we add the condition that all points in the first domain of imprimitivity are actually moved, and only ask whether the action on these other points is actually mapped onto the same cycle under $\pi$. We therefore obtain the upper bound

$$
\frac{(a-1) !^{p-1}}{(k-p-a+1)(k-p-a) \cdots(k-p a+1)}=\frac{(a-1) !^{p-1}(k-p a) !}{(k-p-a+1) !} .
$$

It suffices to show that the probability in question is $\leq \frac{1}{k}$. Compare the $p-1$ largest factors in the numerator, that is, $a^{p-1}$ with the $p-1$ largest factors in the denominator, that is, $(k-p-a+1) \cdots(k-a-2 p+3)$, and note that $k=a b$. Comparing these terms we obtain a contribution $<(b-1-2 p / a)^{1-p}$, continuing 
in this way we find that the probability in question is at most

$$
((b-1-2 p / a)(b-2-2 p / a) \cdots(b-a-2 p / a))^{1-p} .
$$

All factors are at most 1 , thus, to give an upper bound, we may single out any factor easily dealt with. If $b \geq 2 a+4 p / a$, there is one factor $\leq a^{-1}$, and two factors $\leq 2 / b$, hence, the whole product is less than $4 /\left(a b^{2}\right) \leq 1 /(a b)$. Next, note that there are $p-1$ factors which are less than $\frac{1}{2}$, thus, if $2^{p}-1>a b$, we are done. In view of the previous result we see that we may assume $p \leq a$, provided that $a \geq 9$. Again, these special cases can be excluded by looking at the original expression; we therefore obtain the restrictions $b<2 a+4, p<a$.

We now go back to the original fraction and note that in the denominator, there are $(p-1)(a-1)$ consecutive positive integers, hence, we obtain the upper bound

$$
\frac{(a-1) !^{p-1}}{(a p-a-p+1) !} .
$$

This expression is decreasing as $p$ increases, for $p=3$ it becomes

$$
\frac{(a-1) !^{2}}{(2 a-2) !}=\left(\begin{array}{c}
2 a-2 \\
a-1
\end{array}\right)^{-1}<\frac{1}{2 a^{2}+4 a}<\frac{1}{a b},
$$

provided that $a \geq 6$, the remaining cases have to be checked individually again. Hence, we may assume $p=2$, and the probability in question is equal to

$$
\frac{(a-1) !}{(a b-a-1) \cdots(a b-2 a+1)} \leq(b-1)^{1-a},
$$

which is $\leq \frac{1}{a b}$ unless $b=2, b=3$ and $a \leq 6, b=4$ and $a \leq 4$, or $a=2$. However, the special cases dealt with at the beginning imply that $a, b \geq 3$, and we are again lead to a few special cases which are easily excluded separately.

Hence, for imprimitive $H$ we always have $\frac{\mid[x] \cap H}{|[x]|} \leq \frac{k-2 \ell-1}{k}$, and we do not have to consider such groups any more.

To deal with primitive subgroups, we use the following.

Lemma 4. Let $H<S_{k}$ be a primitive subgroup, and suppose that one of the following two conditions hold true.

(1) $H$ contains a $q$-cycle for some prime number $q<k-4$;

(2) $|H|>3^{k}$ or $|H|>2^{k}$ and $k \geq 25$.

Then $H$ is $A_{n}$ or $S_{n}$.

Proof. The first condition is due to (confer [2, II. Satz 4.5]), whereas the second was proven by Maróti[3].

Suppose that $H<S_{n}$ is primitive, not equal to $S_{n}$ or $A_{n}$, and that $\frac{|[x] \cap H|}{|[x]|} \geq$ $\frac{k-\ell p-1}{k}$. Define an equivalence relation on $[x]$ as follows: Two elements $y, z$ are equivalent, if the $\ell$ sets of $p$ points in the $p$-cycles of $y$ and $z$ are equal, and if the action of $y$ and $z$ is the same on each of these sets with the exception of the one containing the least number moved by $y$ and $z$, and on this set, there is some $i$ such that the action of $y^{i}$ is equal to the action of $z$; that is, $y$ and $z$ share $\ell-1 p$-cycles, and move the same points, and on the $p$ points on which they differ, one is a power of the other. Suppose that $\frac{k-\ell p-1}{k}>\frac{1}{p-1}$. Since every equivalence class contains $p-1$ elements of $[x]$, there is one class containing at least 2 elements of $[x] \cap H$. However, their quotient is a single $p$-cycle, thus $H$ contains a $p$-cycle, which is only 
possible for $p \geq k-4$, contradicting our assumption for $k \geq 7$. Hence, we either have $k \leq 6$ or $\frac{k-\ell p-1}{k} \leq \frac{1}{p-1}$. For $p=2$ this argument does not apply, while for $p=3,5$ it is not very efficient. For these cases we argue as follows. In $S_{5}$, the only even permutations which do not consist of a single cycle of prime length are the identity and the permutations conjugate to (12)(34). We call a set $A$ of elements in $S_{5}$ admissible, if the subgroup generated by all elements $\sigma \tau^{-1}, \sigma, \tau \in A$ are of this form, that is, if there is some conjugate of the Klein four group containing all elements $\sigma \tau^{-1}$. By direct inspection we find that an admissible set consisting of elements conjugate to $(12)(34)$ contains at most 3 elements, an admissible set of 3 -cycles contains at most 4 elements, and an admissible set of 5 -cycles contains at most 2 elements. We now form subsets of $[x]$ in the following way: For $p=2$ we choose 5 points $\left\{a_{1}, \ldots, a_{5}\right\}$, and $\ell-2$ pairs of points $\left(x_{1}, y_{1}\right), \ldots,\left(x_{\ell-2}, y_{\ell-2}\right)$, such that all points $a_{i}, x_{i}, y_{i}$ are distinct, and consider the set of all permutations which interchange $x_{i}$ and $y_{i}$, and act as the product of two transpositions on $\left\{a_{1}, \ldots, a_{5}\right\}$. Similarly, for $p=3,5$ we choose five points and $\ell-1$ cycles of length $p$, and form the set of all permutations which act as prescribed on the $\ell-1$ cycles, and as an arbitrary cycle of length $p$ on $\left\{a_{1}, \ldots, a_{5}\right\}$. We do so for all choices of the five points and the cycles. The number $N$ of elements in one subset equals the number of permutations conjugate to $(12)(34),(123)$ or (12345), respectively, in $S_{5}$, that is, it equals 15,20 , or 24 . On average, the proportion of elements contained in $H$ among all elements in a subset is the same as the proportion of elements in $H$ among all of $[x]$, thus, there exists some subset containing at least $\frac{|[x] \cap H|}{|[x]|} \cdot N$ elements in $H$. The quotient of two elements in one subset moves at most 5 points, and if this quotient acts as a cycle of prime length, we obtain $k \leq 9$ or that $H$ is one of $A_{n}, S_{n}$. In other words, if $H \neq A_{n}, S_{n}$, the restriction of the action of a subset to the five-point set is admissible, and using the bounds for the size of admissible sets we obtain $\frac{|[x] \cap H|}{|[x]|} \leq \frac{1}{5}$ for $p=2,3$, and $\frac{|[x] \cap H|}{|[x]|} \leq \frac{1}{12}$ for $p=5$, and therefore the same bounds for $\frac{k-\ell p-1}{k}$.

Now suppose that $k>24$. Then we have $|H| \leq 2^{k}$, and $|[x] \cap H| \geq \frac{|[x]|}{k}$. As a function of $\ell,|[x]|$ is unimodal, thus we have

$$
|[x]| \geq \min \left(\frac{k !}{\left(k-p \ell_{0}\right) ! \ell_{0} ! p^{\ell_{0}}}, \frac{k !}{(k-p\lfloor k / p\rfloor) !\lfloor k / p\rfloor ! p\lfloor k / p\rfloor}\right),
$$

where

$$
\ell_{0}= \begin{cases}\left\lceil\frac{(p-2) k-p+1}{p(p-1)}\right\rceil, & p \geq 7, \\ \left\lceil\frac{11 k}{12}\right\rceil-1, & p=5 \\ \left\lceil\frac{4 k}{5}\right\rceil-1, & p=2,3\end{cases}
$$

is the lower bound for $\ell$ obtained above. We therefore obtain the inequality

$2^{k} \geq|H| \geq|[x] \cap H| \geq \frac{|[x]|}{k} \geq \frac{1}{k} \min \left(\frac{k !}{\left(k-p \ell_{0}\right) ! \ell_{0} ! p^{\ell_{0}}}, \frac{k !}{(k-p\lfloor k / p\rfloor) !\lfloor k / p\rfloor ! p\lfloor k / p\rfloor}\right)$,

and a simple computation shows that this inequality does not hold true for any $k \geq 25$.

Finally, for $k \leq 50$ the primitive groups were tabulated by Sims[11] and are readily available under a computer algebra system such as GAP[1]. There are 154 primitive groups of degree $\leq 24$ which have to be checked individually. This work is feasible, since among these groups there are 45 symmetric or alternating groups, 
and most other groups can be discarded using the trivial estimate $|[\pi] \cap G| \leq G$. There remain only the cases $C_{2} \ltimes C_{5}$ acting on 5 points, and $P S L_{3}\left(\mathbb{F}_{2}\right)$ acting on 7 points, which lead to the exceptions mentioned in the theorem.

\section{Computational examples}

In this section we prove Propositions 3 and 4, beginning with the former.

The following table lists the subgroups of $S_{3}$ together with the number of fixed points of (12) and (123) under the coset action.

\begin{tabular}{c|c|c|c} 
Generators & Order & Fixpoints of (12) & Fixpoints of $(123)$ \\
\hline$(12),(13)$ & 6 & 1 & 1 \\
$(123)$ & 3 & 0 & 2 \\
$(12)$ & 2 & 1 & 0 \\
$(13)$ & 2 & 1 & 0 \\
$(23)$ & 2 & 1 & 0 \\
id & 1 & 0 & 0
\end{tabular}

Similarly, for $D_{10}$, denoting by $\tau_{1}, \ldots, \tau_{5}$ the reflections, we have the following.

\begin{tabular}{c|c|c|c} 
Generators & Order & Fixpoints of $\tau_{1}$ & Fixpoints of $\tau_{2}$ \\
\hline$\tau_{1}, \tau_{2}$ & 10 & 1 & 1 \\
$\tau_{1} \tau_{2}$ & 5 & 0 & 0 \\
$\tau_{1}$ & 2 & 1 & 1 \\
$\tau_{2}$ & 2 & 1 & 1 \\
$\tau_{3}$ & 2 & 1 & 1 \\
$\tau_{4}$ & 2 & 1 & 1 \\
$\tau_{5}$ & 2 & 1 & 1 \\
id & 1 & 0 & 0
\end{tabular}

Denote by $x_{1}, x_{2}, x_{4}$ the number of orbits, on which one symmetric group acts similar to the action on the cosets of $S_{3}, A_{3}$, and 1 , respectively, and let $x_{3}$ be the number of orbits on which the action is similar to one of the coset actions of one of the 2-element subgroups. Similarly, denote by $y_{1}, \ldots, y_{4}$ the number of orbits for the other symmetric group. Finally, denote by $z_{1}, z_{2}, z_{4}$ the number of orbits on which the action of $D_{10}$ is similar to the action on the cosets of $D_{10}, C_{5}$ and 1 , respectively, and by $z_{3}$ the number of orbits of size 2 .

Then we obtain the following conditions:

$$
\begin{aligned}
x_{1}, x_{2}, x_{3}, x_{4}, y_{1}, y_{2}, y_{3}, y_{4}, z_{1}, z_{2}, z_{3}, z_{4} & \geq 0 \\
x_{1}+2 x_{2}+3 x_{3}+6 x_{4} & =1 \\
y_{1}+2 y_{2}+3 y_{3}+6 y_{4} & =1 \\
z_{1}+2 z_{2}+5 z_{3}+10 z_{4} & =1 \\
x_{1}+2 x_{2} & =y_{1}+2 y_{2} \\
x_{1}+x_{3} & =z_{1}+z_{3} \\
y_{1}+y_{3} & =z_{1}+z_{3}
\end{aligned}
$$

The linear form to be maximized is

$$
\begin{aligned}
& \ell\left(x_{1}, x_{2}, x_{3}, x_{4}, y_{1}, y_{2}, y_{3}, y_{4}, z_{1}, z_{2}, z_{3}, z_{4}\right)= \\
& \quad-\left(x_{1}+x_{2}+x_{3}+x_{4}+y_{1}+y_{2}+y_{3}+y_{4}+z_{1}+z_{2}+z_{3}+z_{4}\right) \\
& \quad+\left(x_{1}+2 x_{2}+2 x_{3}+3 x_{4}\right)+\left(y_{1}+2 y_{2}+2 y_{3}+3 y_{4}\right)+\left(z_{1}+2 z_{2}+3 z_{3}+5 z_{4}\right)
\end{aligned}
$$


Since the domain described by the equations and inequalities is convex, and this domain as well a sthe linear form ell is symmetric with respect to the reflection $x_{i} \leftrightarrow y_{i}$, we see that the maximum of $\ell$ on this domain is the same as the maximum under the additional constraint $x_{i}=y_{i}$. Therefore, we obtain the new system

$$
\begin{aligned}
x_{1}, x_{2}, x_{3}, x_{4}, z_{1}, z_{2}, z_{3}, z_{4} & \geq 0 \\
x_{1}+2 x_{2}+3 x_{3}+6 x_{4} & =1 \\
z_{1}+2 z_{2}+5 z_{3}+10 z_{4} & =1 \\
x_{1}+x_{3} & =z_{1}+z_{3}
\end{aligned}
$$

with linear form

$$
\ell^{\prime}\left(x_{1}, x_{2}, x_{3}, x_{4}, z_{1}, z_{2}, z_{3}, z_{4}\right)=2 x_{2}+2 x_{3}+4 x_{4}+z_{2}+2 z_{3}+4 z_{4}
$$

Increasing $x_{2}$ by $3 \epsilon$, and decreasing $x_{4}$ by $\epsilon$ does not change the conditions, but increases the value of $\ell^{\prime}$ by $2 \epsilon$, hence, in the optimum we have $x_{4}=0$, and similarly we deduce $z_{4}=0$. If $x_{1}$ and $x_{2}$ are increased by $\epsilon$, and $x_{3}$ is decreased by $\epsilon$, the equations remain valid, and the linear form is not changed, thus, we may assume that $x_{3}=0$. Similarly, if we increase $z_{1}$ by $\epsilon, z_{2}$ by $2 \epsilon$ and decrease $z_{3}$ by $\epsilon$, the value of $\ell^{\prime}$ does not change, and we obtain $z_{3}=0$, thus, $x_{1}=z_{1}$. We therefore obtain the system

$$
\begin{aligned}
& x_{1}+2 x_{2}=1 \\
& x_{1}+2 z_{2}=1
\end{aligned}
$$

with linear form

$$
\ell^{\prime \prime}\left(x_{1}, x_{2}, z_{2}\right)=2 x_{2}+z_{2}
$$

which has obviously the optimal value $\mu=\frac{3}{2}$.

We now compute $\mu\left(S_{4} *_{C_{2} \times C_{2}} S_{4}\right)$ with amalgamation as described in Proposition 4.

We first give a complete list of subgroups of $S_{4}$ together with the induced actions of the two subgroups. Let $\tau_{1}, \ldots, \tau_{5}$ be the transitive actions of $C_{2} \times C_{2}$, where $\tau_{1}$ is trivial, $\tau_{5}$ is regular, $\tau_{2}$ is the action on the cosets of $\{1,(12)(34)\}, \tau_{3}$ corresponds to $\{1,(13)(24)\}$ respectively $\{1,(12)\}$, and $\tau_{4}$ corresponds to $\{1,(14)(23)\}$ respectively $\{1,(34)\}$. We can simplify our computations, since we are only dealing with one vertex group, it is easy to see that in this case the induced action only depends on the conjugacy class of the subgroup, not on the subgroup itself. Thus, we only have to consider 11 distinct representations of $S_{4}$, the relevant information is contained in the following table. 


\begin{tabular}{c|c|c|c|c|c|c|c|c|c|c|c|c|c} 
& Subgroup & Index & \multicolumn{3}{|c}{ Representation of $U_{1}$} & \multicolumn{6}{|c}{ Representation of $U_{2}$} \\
\hline & (Name or generators) & & $\tau_{1}$ & $\tau_{2}$ & $\tau_{3}$ & $\tau_{4}$ & $\tau_{5}$ & $\tau_{1}$ & $\tau_{2}$ & $\tau_{3}$ & $\tau_{4}$ & $\tau_{5}$ \\
\hline$\rho_{1}$ & $S_{4}$ & 1 & 1 & 0 & 0 & 0 & 0 & 1 & 0 & 0 & 0 & 0 \\
$\rho_{2}$ & $A_{4}$ & 2 & 2 & 0 & 0 & 0 & 0 & 0 & 1 & 0 & 0 & 0 \\
$\rho_{3}$ & $(12),(13)(24)$ & 3 & 3 & 0 & 0 & 0 & 0 & 1 & 1 & 0 & 0 & 0 \\
$\rho_{4}$ & $(12)(34),(13)(24)$ & 6 & 6 & 0 & 0 & 0 & 0 & 0 & 3 & 0 & 0 & 0 \\
$\rho_{5}$ & $(12),(123)$ & 4 & 0 & 0 & 0 & 0 & 1 & 0 & 0 & 1 & 1 & 0 \\
$\rho_{6}$ & $(1234)$ & 6 & 0 & 1 & 1 & 1 & 0 & 0 & 1 & 0 & 0 & 1 \\
$\rho_{7}$ & $(12),(34)$ & 6 & 0 & 1 & 1 & 1 & 0 & 2 & 0 & 0 & 0 & 1 \\
$\rho_{8}$ & $(123)$ & 8 & 0 & 0 & 0 & 0 & 2 & 0 & 0 & 0 & 0 & 2 \\
$\rho_{9}$ & $(12)(34)$ & 12 & 0 & 2 & 2 & 2 & 0 & 0 & 2 & 0 & 0 & 2 \\
$\rho_{10}$ & $(12)$ & 12 & 0 & 0 & 0 & 0 & 6 & 0 & 0 & 2 & 2 & 1 \\
$\rho_{11}$ & id & 24 & 0 & 0 & 0 & 0 & 6 & 0 & 0 & 0 & 0 & 6 \\
\hline
\end{tabular}

From these table we find that our linear problem has 22 variables $x_{1}, \ldots, x_{11}, y_{1} \ldots, y_{11}$, corresponding to the 11 representation of each factor, and the equations

$$
\begin{aligned}
\sum_{i=1}^{11} n_{i} x_{i} & =1 \\
\sum_{i=1}^{11} n_{i} y_{i} & =1 \\
x_{1}+2 x_{2}+3 x_{3}+6 x_{4} & =y_{1}+y_{3}+2 y_{7} \\
x_{6}+x_{7}+2 x_{9} & =y_{2}+y_{3}+3 y_{4}+y_{6}+2 y_{9} \\
x_{6}+x_{7}+2 x_{9} & =y_{5}+2 y_{10} \\
x_{5}+2 x_{8}+6 x_{10}+6 x_{11} & =y_{6}+y_{7}+2 y_{8}+2 y_{9}+y_{10}+6 y_{11}
\end{aligned}
$$

where $n_{i}$ is the number of points $\rho_{i}$ acts upon. The linear form to be optimized is

$$
\begin{gathered}
\ell\left(x_{1}, \ldots, x_{11}, y_{1}, \ldots, y_{11}\right)=-\sum_{i=1}^{11} x_{i}-\sum_{i=1}^{11} y_{i}+ \\
x_{1}+2 x_{2}+3 x_{3}+6 x_{4}+x_{5}+2 x_{6}+2 x_{7}+2 x_{8}+4 x_{9}+6 x_{10}+6 x_{11} .
\end{gathered}
$$

Solving this linear program we find that the optimum is attained in the point $x_{4}=\frac{1}{18}, x_{11}=\frac{1}{36}, y_{7}=\frac{1}{6}$, and all other variables equal to 0 . The value of the form in this point is $\frac{1}{4}$, thus, we find $\mu(\Gamma)=\frac{1}{4}$.

The computation of the Euler characteristics is straightforward in both cases.

Note that in the second example the optimising tuple contains two non-zero values $x_{i}$, that is, different from the case of free products of finite groups it is not true that for almost all homomorphisms $\varphi: \Gamma \rightarrow S_{n}$ the restriction to a finite subgroup $U$ decomposes into orbits on almost all of which $U$ acts in the same way. This observation makes the existence of a simple relation between $\mu(\Gamma)$ and group theoretic data of $\Gamma$ rather unlikely.

\section{REFERENCES}

[1] The GAP Group, GAP - Groups, Algorithms, and Programming, Version 4.4; 2006. (http://www.gap-system.org)

[2] B. Huppert, Endliche Gruppen, Springer-Verlag Berlin Heidelberg New York 1967.

[3] A. Maróti, On the orders of primitive groups, J. Algebra 258 (2002), 631-640. 
[4] T. W. Müller, Combinatorial aspects of finitely generated virtually free groups, J. London Math. Soc. (2) 44 (1991), 75-94.

[5] T. W. Müller, Subgroup growth of free products, Invent. Math. 126 (1996), 111-131.

[6] T. W. Müller, J.-C. Schlage-Puchta, Character theory of symmetric groups, subgroup growth of Fuchsian groups, and random walks, Adv. Math. 213(2007), 919-982

[7] T. W. Müller, J.-C. Schlage-Puchta, Decomposition of the conjugacy representation of the symmetric group, and subgroup growth, submitted.

[8] T. W. Müller, J.-C. Schlage-Puchta, Some examples in the theory of subgroup growth, Monatsh. Math. 146 (2005), 49-76.

[9] T. W. Müller, J.-C. Schlage-Puchta, Some probabilistic subgruops of a finitely generated group, in preparation.

[10] J.-P. Serre, Trees, Springer-Verlag, Berlin-New York, 1980.

[11] C. C. Sims, Primitive groups, graphs and block designs, Ann. New York Acad. Sci. 175 (1970), 351-353. 